\title{
Theaterpolitik als Kulturpolitik
}

\author{
Max Fuchs
}

\section{Zusammenfassung}

Theaterpolitik ist ein Teil der Kulturpolitik. Das heißt, dass man Strukturmerkmale der Kulturpolitik auf die Theaterpolitik übertragen kann. Das gilt insbesondere für den Governance-Ansatz. Hierbei geht es darum, dass man die verschiedensten Akteure bei der Politikgestaltung - und dies auf den unterschiedlichen Ebenen (Kommune, Land, Bund, Europäische Union, UNESCO) und in den unterschiedlichen Politikfeldern (neben Kulturpolitik sind es Rechtspolitik, Sozialpolitik, Wirtschaftspolitik, Kommunalpolitik etc.) - unterscheiden kann. Selbst eine grobe Sichtung dieser Vielfalt an Zugangsmöglichkeiten zeigt, dass man mit sehr unterschiedlichen Verständnis- und Begründungsweisen von Kulturpolitik rechnen muss.

\section{Schlüsselwörter}

Theaterpolitik • Kulturförderung • Cultural Governance • Entwicklung des Theaters $\bullet$ Kulturpolitische Leitmotive

M. Fuchs $(\bowtie)$

Institut für Erziehungswissenschaft, Universität Duisburg-Essen, Wuppertal, Deutschland E-Mail: maxfuchs@web.de 


\section{1 Überlegungen zu einer systematischen Annäherung an die Theaterpolitik}

Die Theaterpolitik ist ein Teilbereich der Kulturpolitik. Das bedeutet unter anderem, dass man sie ähnlich strukturieren kann wie die Kulturpolitik. Das Problem hierbei besteht allerdings darin, dass es zwar eine Fülle kulturpolitisch zu verstehender Debatten und Diskurse gibt, dass es aber zu einer systematischeren Theorienbildung bislang kaum gekommen ist. So finden sich kaum Ansätze, die politische Theorien der Gegenwart (Brodocz und Schaal 2017) auf den Theaterbereich übertragen. Zwar gibt es Ansätze zu einer Kulturpolitikforschung, etwa im Kontext des Instituts für Kulturpolitik an der Universität Hildesheim bzw. im Rahmen des Instituts für Kulturpolitik der Kulturpolitischen Gesellschaft, doch handelt es sich hierbei oft um Spezialfragen, deren Bearbeitung sicherlich hilfreich und notwendig ist: Von einer Theorienbildung in anspruchsvollerem Sinne kann man allerdings kaum sprechen. Dies zeigt sich auch, wenn man nach einer einschlägigen Fachliteratur sucht. So gibt es zwar eine ganze Reihe von - zum Teil hoch reflektierten - Konzeptvorschlägen darüber, was man unter einer entsprechend engagierten Kulturpolitik verstehen kann, doch gibt es relativ wenig Fachliteratur, die einen Strukturierungsversuch unternimmt (siehe etwa Klein 2005; Fuchs 1998, 2007). Zu den engagierten Konzeptvorschlägen gehören sicherlich die beiden Ansätze von Hilmar Hoffmann („Kultur für alle“; 1979) und von Hermann Glaser („Bürgerrecht Kultur“; 1974/1983). Auch das Buch von Oliver Scheytt (2008) über den „Kulturstaat Deutschland“ kann man in diese Reihe stellen.

Es gibt zudem bei den verschiedenen Parteien kulturpolitische Konzeptvorschläge und Zielformulierungen und kulturpolitische Ziele in Koalitionsverträgen. Ein wichtiges Dokument in diesem Zusammenhang ist der Schlussbericht der Enquete-Kommission des Deutschen Bundestages „Kultur in Deutschland“ (2008), in dem umfassend eine Gesamtdarstellung kultureller Angebote in Deutschland unter Einbeziehung der Kultur- und Kreativwirtschaft unter einer europäischen und internationalen Perspektive vorgestellt wird. Dieses Dokument dürfte immer noch die ausführlichste und ambitionierteste Gesamtdarstellung sein.

Kulturpolitische Konzeptionen findet man zudem auf Ebene der Bundesländer und der Kommunen, etwa im Rahmen von „Kulturentwicklungsplänen“. Bei Letzteren gibt es inzwischen sogar eine gewisse Tradition, entsprechende Konzeptpapiere unter Beteiligung der Zivilgesellschaft zu entwickeln. Beispiele sind entsprechende Konsultationsprojekte etwa in Thüringen oder Baden-Württemberg. Nicht zu vergessen sind konzeptionelle Überlegungen zur Kulturpolitik und zur 
Rolle des Kulturellen, der Künste und der Medien in übernationalen Zusammenschlüssen wie etwa der Europäischen Union (hier sogar mit einem Haushaltsetat), dem Europarat, der insbesondere in der Geschichte der 1960er und 1970er Jahre eine wichtige Rolle als Vordenker und Impulsgeber gespielt hat, und der UNESCO mit ihrer spezifischen Verantwortlichkeit für Fragen der Erziehung, der Kultur und der Wissenschaften (Schwencke 2006). In diesem Kontext ist etwa an die ,Weltdekade zur kulturellen Entwicklung“ und an entsprechende internationale Fachkonferenzen wie etwa die Abschlusskonferenz in Stockholm $1998 \mathrm{zu}$ erinnern. Zu Teilbereichen der Kultur- und Bildungspolitik haben sich zudem eigenständige Diskurse entwickelt, etwa zu der Frage der künstlerischen und kulturellen Bildung mit inzwischen zwei Weltkonferenzen der UNESCO 2006 in Lissabon und 2010 in Seoul, bei denen ebenfalls programmatische Papiere zur Weiterentwicklung der kulturellen Bildung erarbeitet wurden (siehe auf der Homepage der Deutschen UNESCO-Kommission, www.unesco.de, die beiden Abschlussdokumente „Lissabon Roadmap“ und „Seoul-Agenda“; Deutsche UNESCO-Kommission 2007).

In der kulturpolitischen Diskussion, die einen wissenschaftlichen Anspruch erhebt, sind inzwischen zumindest zwei Ansätze aus der allgemeinen politikwissenschaftlichen Diskussion übernommen worden. Zum einen handelt es sich hier um die Übertragung des Governance-Ansatzes auf die Kulturpolitik (,Cultural Governance"), zum anderen geht es um die Aufgliederung des Gesamtfeldes der Politik in die drei Bereiche policy, polity und politics.

Bei dem Governance-Ansatz sind zwei Aspekte zu beachten, die auch gerade in der Kulturpolitik wichtig sind: dass nämlich Politik auf mehreren Ebenen verhandelt wird, nämlich auf der kommunalen, der Länder-, der Bundes-, der europäischen und der internationalen Ebene. Dabei sind die Aufgabenstellungen, die in der jeweiligen Ebene verhandelt werden, nicht identisch. Man kann des Weiteren die oben genannte Dreiteilung unterscheiden, nämlich policy (als Thematisierung der inhaltlichen Dimension von Kulturpolitik, also etwa die Frage der Konzepte und Ziele), polity (als System der Institutionen, der formalen und strukturellen Gegebenheiten) und politics (als das alltägliche Geschäft politischer Aushandlungsprozesse zwischen den beteiligten Akteuren).

Wenn man sich - etwa in einer Tageszeitung - darüber informiert, welche kulturpolitischen Themen diskutiert werden, so wird man etwas von Finanznöten von Kultureinrichtungen lesen können, man wird über aufregende neue Inszenierungen, Choreografien, Ausstellungen oder literarische Neuerscheinungen informiert. Nicht zuletzt wird es um Personalfragen gehen, also etwa um die Besetzung von Leitungsfunktionen von Kultureinrichtungen bzw. darum, wer welchen der zahlreichen Kulturpreise bekommen hat. Gelegentlich wird man sicherlich auch 
Informationen darüber erhalten, wie die Entwicklung des - vor allem kommunalen - Kulturetats verläuft oder welche programmatischen Vorstellungen ein neuer Kulturdezernent oder eine neue Kulturministerin hat.

Ordnet man diese Themen den verschiedenen Ebenen unseres politischen Gemeinwesens zu, dann ist es in erster Linie die kommunale Ebene, in der diese Fragen diskutiert und auch entschieden werden. Dies deckt sich mit dem System der Kulturförderung in Deutschland, bei denen der Bund nur einen etwa zehnprozentigen Anteil hat und der Rest zwischen Ländern und Kommunen aufgeteilt wird (man spricht von einem ,kooperativen Kulturföderalismus“). Dies gilt insbesondere auch für das Theater, denn dies ist der Bereich, der den größten Anteil an der öffentlichen Kulturförderung in Deutschland bekommt. Es geht also im Wesentlichen um Personal- und Förderfragen, die vor allem auf kommunaler und in Teilen auch auf Landesebene angesiedelt sind.

Der Bund hat aufgrund der schlechten Erfahrungen mit einer zentralistischen politischen Ordnung gemäß dem deutschen Grundgesetz nur die Aufgaben, die ihm das Grundgesetz explizit zuspricht („Kulturhoheit der Länder"). Allerdings bleibt eine ganze Reihe von Aufgaben übrig, für die der Bund Verantwortung hat. Es geht hierbei im Wesentlichen um die Gestaltung von Rahmenbedingungen. Diese sind zwar von existenzieller Bedeutung im Kulturbereich, doch haben die Kommunen und zu einem großen Teil auch die Länder kaum Mitspracherechte. So geht es etwa um das Urheberrecht, wobei sich hier die zentrale Kompetenz inzwischen auf die Ebene der Europäischen Union verlagert hat. Es geht um Fragen der sozialen Absicherung von Künstler*innen (Künstlersozialkasse mit der Möglichkeit einer Krankenversicherung und einer Ansparung einer Rentenversorgung). Der Bund muss auch die nationalen Interessen auf der Ebene der Europäischen Union einbringen, wo nicht nur die zentrale Kompetenz für das Urheberrecht liegt, sondern wo auch wichtige Fragen der Mobilität von Künstler*innen bzw. von Kunstwerken ausgehandelt werden (Visa-Erteilung, Zollprobleme, Arbeitsgenehmigungen etc.).

Die beiden oben genannten internationalen Organisationen wie Europarat und UNESCO haben weder eine gesetzgeberische noch eine finanzielle Bedeutung. Allerdings hat der Europarat durch wichtige Konzeptdiskussionen in den 1960er und 1970er Jahren zu einem Paradigmenwechsel im Verständnis der Kulturpolitik geführt, als er etwa - quasi als Vorläufer einer in Deutschland sogenannten „Neuen Kulturpolitik“ - Ideen einer soziokulturellen Politik und Konzepte wie „Demokratisierung der Kultur“ bzw. „kulturelle Demokratie“ vorschlug (siehe dazu die entsprechenden Dokumente in Schwencke 2006). Ähnliches gilt für die UNESCO, die 1982 eine erste Weltkonferenz zur Kulturpolitik in Mexiko City durchführte, in der man sich etwa auf einen Begriff von Kultur geeinigt hat, der 
sowohl die künstlerischen Aktivitäten erfasste, der aber auch die Lebensweise und die Werte und Normen in der Gesellschaft einbezog. Europarat und UNESCO sind daher für alle inhaltlichen Argumentationen immer auch wichtige Referenzquellen, auf die man Bezug nehmen kann. Dies gilt auch für die aktuelle Debatte über Teilhabe, denn dies ist ein Kernbegriff in dem System der unterschiedlichen Menschenrechte und er ist gerade im Hinblick auf die im ersten Abschnitt vorgestellte Problematik einer mangelhaften Beteiligung aller gesellschaftlichen Gruppen an der Nutzung der kulturellen Angebote unserer Kultureinrichtungen und insbesondere des Theaters von großer Bedeutung.

Auf den Ebenen politischer Gestaltung sind hierzulande in erster Linie staatliche Akteure bzw. Akteure der öffentlichen Hand aktiv. Dies bezieht sich auf die jeweiligen Parlamente (Stadtrat, Landtage, Bundestag) sowie auf die Exekutive und Verwaltung (Kulturdezernent*in und Oberbürgermeister*in, Kulturministerien). Auf Bundesebene ist zudem die Kultusministerkonferenz als Arbeitsgemeinschaft der Länder zu nennen. Immer schon gab es allerdings zivilgesellschaftliche Vertretungen in Gremien der unterschiedlichen Arbeitsfelder, die sich an den Diskussionen und Diskursen beteiligt haben und für die auf den verschiedenen Ebenen auch offizielle Mitwirkungsmöglichkeiten geschaffen wurden.

Diese Einfluss- und Mitwirkungsmöglichkeiten beziehen sich zum einen auf öffentliche Medien, die nicht unwichtig sind, wenn es darum geht, Argumentationen öffentlich zu machen. Hier hat das Theater immer schon gute Chancen gehabt, seine Interessen zu artikulieren. Denn das Theater war - etwa über die Theaterkritik - schon immer von besonderem Interesse für das Feuilleton. Es gibt aber auch institutionalisierte Formen zur Einbindung der zivilgesellschaftlichen Organisationen. So gibt es Zusammenschlüsse und Interessenverbände der Theater auf allen Ebenen, die wiederum in entsprechenden Dachorganisationen im Kulturbereich (Landeskulturräte, Deutscher Kulturrat) vertreten sind. Der Deutsche Bühnenverein hat zudem einen ständigen Sitz im Kulturausschuss des Deutschen Städtetages.

Dabei wird man sehen, dass zwar auf jeder Ebene und bei jedem Träger inhaltliche Argumentationen und Diskussionen über die generelle Rolle des Theater zu finden sind (Begründung und Legitimation), dass sich jedoch die Kultur- und Theaterpolitik auf jeder der Ebenen in der Aufgabenstellung sehr unterschiedlich gestaltet. So geht es auf kommunaler Ebene sehr stark um Personalfragen und Fragen der Förderung. Diese spielt auf Landesebene ebenfalls eine Rolle, doch hätte das Land aufgrund unserer grundgesetzlichen Regelung zusätzlich eine Gesetzgebungskompetenz, etwa durch die Verabschiedung eines generellen Kultur- oder eines speziellen Theaterfördergesetzes. Solche Forderungen nach 
gesetzlichen Regelungen werden seit langem vom Kulturbereich erhoben, allerdings nur in Ausnahmefällen umgesetzt (z. B. im Sächsischen Kulturraum- sowie im NRW-Kulturfördergesetz).

Auf Bundesebene spielt eine Regelförderung quasi keine Rolle. Allerdings kann die Bundeskulturstiftung durch eine Projektförderung wichtige Impulse geben, wie etwa durch das frühere Projekt „Heimspiel“, in dem solche Theateraktivitäten gefördert wurden (vgl. Beitrag in diesem Band), in denen das Theater bewusst seine Rolle wahrgenommen hat, Probleme der jeweiligen Stadt aufzugreifen und öffentlich zu diskutieren. Die zentrale Aufgabe der Kulturpolitik auf Bundesebene besteht allerdings darin, gesetzliche Rahmenbedingungen für den Kulturbereich zu schaffen. Das betrifft etwa das Urheberrecht, Fragen der Besteuerung oder rechtliche Regelungen, die für die soziale Lage der Künstler*innen relevant sind (Künstlersozialkasse). Dies bedeutet allerdings auch, dass sich eine effektive Kultur- und Theaterpolitik sehr stark sachkundig in anderen Politikfeldern bewegen muss. So liegt die Kompetenz für Steuerfragen im Finanzministerium, das Problem des Urheberrechts liegt beim Justizministerium und Fragen der sozialen Absicherung ressortieren im Sozialministerium. Geht es zudem um Fragen des internationalen Kulturaustauschs, die auch für das Theater relevant sind, ist zusätzlich die Auswärtige Kultur- und Bildungspolitik (AKB) zu berücksichtigen.

\section{$2 \quad$ Entwicklungen und Diskurse}

Man kann die Geschichte des Theaters als Geschichte von Krisen und Reformforderungen schreiben. Dies gilt zumindest für die letzten 250 Jahre, und es gilt speziell für Deutschland, wo das Theater über lange Zeit das kulturelle Leitmedium war (Fischer-Lichte 1993; Nipperdey 1998, S. 793 ff.). Nun klingt diese Behauptung dramatischer, als es ein Blick auf die gesellschaftlichen Realitäten bestätigen kann. So muss man als erstes differenzieren, was mit „Theater“ gemeint ist: Ist die künstlerische Ausdrucksform Theater damit gemeint, so muss man feststellen, dass die genannte Behauptung auch für die anderen Kunstsparten wie Musik oder bildende Kunst Gültigkeit hat, sodass die konflikthaltige Entwicklungslogik des Theaters zumindest kein Alleinstellungsmerkmal ist. Dies gilt insbesondere für Prozesse der Autonomisierung und der Emanzipation der Kunstfelder, beim Theater: die Emanzipation der lange Zeit verbreiteten Meinung, dass es sich dabei um eine eher sekundäre Umsetzung einer eigentlich 
wichtigen literarischen Vorlage handelt (Fischer-Lichte 1993). Dieser Prozess findet gleichermaßen in der Musik und in der bildenden Kunst mit einer besonderen Dynamik erst im 19. Jahrhundert statt (Fuchs 2011a).

Wenn man damit das Theater als Institution und als System von Theaterhäusern begreift, dann wird man auf die Frage eingehen müssen, wer diese Einrichtungen finanziert und aus welchen Gründen dies geschieht. Insbesondere wird man dann erkennen, dass es hier - ebenfalls im 19. Jahrhundert - zu einer Verlagerung der Trägergruppe kommt, ganz so, wie es der Titel von Bernd Wagner in seiner verdienstvollen Analyse der Entwicklung der Kulturpolitik in Deutschland formuliert: „Vom Fürstenhof zur Bürgergesellschaft“ (Wagner 2009).

Man wird berücksichtigen müssen, um welche Realisierungsform des Theaters es geht: um das sich allmählich entwickelnde professionelle Theater, um das verbreitete Laienspiel, um das Theater in Großstädten oder im ländlichen Bereich, um das reine Unterhaltungstheater oder um das Theater mit einem hohen künstlerischen Anspruch und nicht zuletzt um die Rolle des Theaterspielens in Bildungsprozessen, vor allem in der Schule. Die Rede vom Theater ist also ausgesprochen komplex, sodass man bereits ohne weitere Vertiefung des Begriffs der Theaterpolitik sowohl von einer hohen Komplexität als auch von einem dynamischen Wandel in der Geschichte ausgehen muss.

Das Theater hat im Hinblick auf die These von einer krisenhaften Entwicklung nicht nur im Bereich der Künste kein Alleinstellungsmerkmal, sondern man kann diese These auch an ganz anderen Gesellschaftsbereichen und Politikfeldern verifizieren. So lässt sich der Verlauf der Bildungs- und Schulpolitik ebenfalls als eine von Krisen und Reformen geprägte Entwicklung beschreiben. Dasselbe gilt letztlich auch für Politikfelder wie etwa die Sozial- oder Wirtschaftspolitik. Dies hängt sicherlich zum einen damit zusammen, dass die moderne Gesellschaft, auf deren Gestaltung die unterschiedlichen Politiken Einfluss nehmen wollen, durch Veränderung, Dynamik und - wie viele früher meinten - durch Fortschritt charakterisiert wird. Es hängt sicherlich aber auch damit zusammen, dass man bei der Formulierung von Reformzielen meistens zu vollmundigen und anspruchsvollen Zielformulierungen neigt, die dann letztlich nicht realisiert werden können. Die Formulierung solcher Ziele dient häufig der Legitimation, also der Begründung und Berechtigung der vorgeschlagenen Veränderungen, für die man um Zustimmung wirbt. Diese Zielformulierungen sind dabei abhängig von Fragen und Problemen, die jeweils in den gesellschaftlichen Diskursen aktuell sind.

Akzeptiert man jedoch die These von der dynamischen Entwicklung moderner Gesellschaften, dann liegt es auf der Hand, dass solche Begründungsmetaphern, 
die oft genug Wirksamkeitsunterstellungen sind, notwendigerweise veralten müssen. Ein gutes Beispiel aus dem Theaterbereich ist der vom Deutschen Bühnenverein vor etwa 20 Jahren propagierte und plakatierte Slogan „Theater muss sein“ (Deutscher Bühnenverein 2003). Lange Zeit konnten die Urheber dieses Slogans davon ausgehen, dass er zumindest bei einer entscheidungsrelevanten Gruppe der Bevölkerung akzeptiert wird. Inzwischen wird seit längerer Zeit auch im Theaterbereich wahrgenommen, dass die Selbstverständlichkeit der Akzeptanz dieses Slogans verloren gegangen ist. So hat der Deutsche Bühnenverein (2003) eine Sammlung von Texten veröffentlicht, in denen prominente Autor*innen, Regisseur*innen und Intendant*innen Antworten auf fünf oft gestellte Fragen geben: 1) Globale Welt, lokale Theater. Was soll das? 2) Zwischen PISA-Studien und Abgesang auf das Bürgertum - Theater, ein historisches Relikt? 3) Gesucht: Ein neues Bild der Städte 4) In Mediengewittern - das Theater überflüssig? 5) Werktreue und Regietheater: Das Theater zwischen Museum und moderner Kunst.

Doch nach wie vor ergeben sich aus dem genannten Slogan wichtige Fragen, die für eine jede Theaterpolitik ergiebig sind. So kann man etwa fragen, ob „Theater muss sein“ eine empirische Aussage über einen zweifelsfreien Tatbestand ist, der dann allerdings auch belegt werden müsste. Man kann diesen Slogan aber auch als normative Aufforderung verstehen, wobei sich hier ebenfalls eine mehrfache Begründungspflicht ergibt. So lassen sich sinnvoll die berühmten W-Fragen stellen, etwa: Wieso muss Theater sein? Für wen muss Theater sein? Welches Theater muss eigentlich sein (angesichts der oben skizzierten Pluralität)? Wo muss es ein solches Theater geben? Wer muss für ein solches Theater die Verantwortung übernehmen?

Angeregt durch diesen Slogan kann man auf einer Suchmaschine im Internet die Worte eingeben „Theater muss...“. Es ist dabei nicht nur interessant, dass man mit einer Fülle von Antworten belohnt wird, auch das Spektrum dieser Antworten ist aufschlussreich. Ohne Vollständigkeit will ich eine Reihe von Beispielen geben (Anfrage auf google.de am 27.01.2020). Danach muss Theater: mit allem rechnen, ein Stück absetzen, vibrieren, saniert werden, politisch sein, unterhalten, weh tun, sich ändern, einen Behindertenhund reinlassen, digitalisiert werden, interaktiv sein, kosmisch sein, Menschen berühren, für Schülerinnen und Schüler und somit für die Schule präsent sein, es muss Schule machen, etwas wert sein, verstehbar bleiben, wie Fußball sein, sich öffnen, Feinschmecker und Vielfraße bedienen.

Offensichtlich beziehen sich einige dieser Zuschreibungen auf das Gebäude (Scheytt et al. 2016). Einige der Formulierungen beziehen sich auf die Erweiterung der Zielgruppen. Einige greifen aktuelle Debatten wie etwa die der Digitalisierung auf und natürlich ist explizit oder implizit immer auch von der 
Notwendigkeit einer angemessenen finanziellen Unterstützung die Rede. Einige Forderungen beziehen sich auf Änderungsnotwendigkeiten der Inhalte des Theaters oder der Art und Weise, wie Theater präsentiert wird und auf erwünschte Wirkungen des Theaterbesuchs. In jeder der genannten Positionen kann man die Ansicht unterstellen, dass auf alle Fälle das Theater nicht so bleiben kann, wie es ist.

Aufgrund der großen Rolle der Kommunen bei der Finanzierung des Theaters in Deutschland hängt die Existenz von Theatern wesentlich von der kommunalen Haushaltssituation ab. Diese ist allerdings seit Jahrzehnten traditionell schlecht, zumal die Kommunen kaum eigene Steuern erheben dürfen und die jeweiligen Bundesländer und der Bund immer wieder Gesetze verabschieden, deren Umsetzung durch die Kommunen erfolgt, wodurch erhebliche kommunale Finanzmittel gebunden werden. Die Finanznot der Kommunen war besonders prekär in den späten 1980er Jahren. In diesem Kontext sollte durch eine Verwaltungsreform und konkret durch die Einführung des sogenannten „Neuen Steuerungsmodells“ die Finanzkrise gelöst oder zumindest abgemildert werden. Es ging dabei um die Übertragung betriebswirtschaftlicher Steuerungsmethoden auf die öffentliche Verwaltung (Behrens et al. 1995). Interessanterweise hatte man im Rahmen der damaligen Kommunalen Stelle zur Verwaltungsvereinfachung (KGSt) die ersten Versuche bei Kultureinrichtungen, nämlich bei Museen und Theatern unternommen (KGSt 1989; Jann 1998).

Dieser Paradigmenwechsel hatte zwar bei weitem nicht die Erfolge, die man sich davon versprach, er hatte aber gravierende Auswirkungen auf die Handlungslogik in der Kulturpolitik: Einzug hielt eine betriebswirtschaftliche Sprache und Denkweise, eingeführt wurden neue Kontrollmechanismen und seitdem bestehen deutliche Einnahmeerwartungen an Kultureinrichtungen. Eine Folge war u. a. die Veränderung der Rechtsformen von Kultureinrichtungen. Auch der Aufschwung des Kulturmanagements in Deutschland steht mit dieser Entwicklung in enger Verbindung.

Eine zweite Problematik ergab sich durch die deutsche Einigung. Weder die Städte in Ostdeutschland noch die neuen Bundesländer waren in der Lage, die reichhaltige kulturelle Infrastruktur der DDR in der bisherigen Form aufrecht zu erhalten. In der Folge kam es zu gravierenden Veränderungen; der Bund musste in erheblichem Umfang finanziell einspringen, sodass sich seitdem der Anteil der Bundesförderung im Bereich der Kultur bis heute nahezu verdreifacht hat.

Anfang des neuen Jahrtausends sprach man erneut von einer Krise des Theaters (Institut für Kulturpolitik der Kulturpolitischen Gesellschaft 2004). Dabei ging es zum einen um Theaterstrukturen und um Finanzierungsfragen, zum anderen aber 
auch um inhaltliche Fragen wie etwa Fragen des Spielplans sowie um die Funktionen, die das Theater in der Stadt übernehmen kann und soll. Der damalige Bundespräsident Johannes Rau forderte ein neues „Bündnis für Theater“, er formulierte allerdings auch Kritikpunkte: „Die Theater können es sich nicht leisten, wenn ganze Bevölkerungsgruppen abseitsstehen,

- weil sie sich Theater nicht leisten können oder

- weil die Welt der Spielpläne ihnen fremd ist,

- weil ihnen wegen ihrer kulturellen Herkunft unser klassisches Repertoire fremd ist und

- weil junge Menschen eher bei den neuen Medien bleiben, wenn die Theater ihnen keine Angebote bereithalten, die bei ihren Interessen ansetzen und so ihr Interesse wecken.“ (Rau 2004, S. 39).

Johannes Rau berief eine Arbeitsgruppe ,Zukunft von Theater und Oper in Deutschland" ein, die in ihrem Zwischenbericht (2004) einen umfassenden Katalog von Funktionszuschreibungen und Zielen des Theaters formulierte, die zum Teil Widersprüche zur Mängelliste des Bundespräsidenten aufweist. Einige Beispiele:

- die Theater sind ein großes historisches Erbe

- das Theater ist ein Ort, an dem nach dem Sinn des Lebens, nach Werten und Orientierungen für das Zusammenleben gefragt und gesucht wird

- das Theater ist Ort der Auseinandersetzung mit der Vergangenheit und zugleich Zukunftswerkstatt

- Theater ist Aufklärung und damit Widerstand gegen das Verdrängen des Vergangenen

- das Theater stellt Alltag dar und stellt ihn auf den Kopf

- Theater stellt Vertrautes infrage und macht uns Fragwürdiges vertraut.

Insgesamt wird das Theater von der Arbeitsgruppe primär als „ein Forum des öffentlichen Diskurses und der öffentlichen Selbstverständigung" verstanden (Arbeitsgruppe „Zukunft von Theater und Oper in Deutschland“ 2004, S. 344). Auch wenn man diese Funktionszuschreibung akzeptiert, so ist doch festzuhalten, dass der entscheidende Punkt, den Johannes Rau in seinen Hinweisen anspricht, nicht reflektiert wird. Doch wenn das alles gilt, was oben aufgeführt wird, und wenn man all dies für existenziell notwendig hält, dann muss man umso mehr darüber nachdenken, wem diese Möglichkeit der Selbstreflexion und Orientierung nicht gegeben wird. Interessanterweise spricht derselbe Zwischenbericht 
ein Problem an, das in der oben zitierten Onlinerecherche nirgends angesprochen, das aber von vielen für gravierend gehalten wird: die Notwendigkeit einer Modernisierung der Theaterstrukturen.

Offensichtlich hat sich in den letzten 15 Jahren hier weniger verändert, als gelegentlich behauptet wird: So nimmt die Schriftstellerin und viel gespielte Theaterautoren Sibylle Berg (2017) in einem Vortrag auf einer Tagung der Intendantengruppe des Deutschen Bühnenvereins am 09.12.2017 die berühmte Spiegel-Metapher insofern ernst, als dass sie dem Theater selbst und insbesondere der Theaterstruktur einen Spiegel vorhält. Ihr Fazit: An der konservativen und hierarchischen Struktur des Theaters hat sich kaum etwas geändert.

\section{Begründungsweisen und Generationsverhältnisse}

Albrecht Göschel, seinerzeit wissenschaftlicher Mitarbeiter des Deutschen Instituts für Urbanistik (difu), dem Forschungsinstitut des Deutschen Städtetages, legte Anfang der 1990er Jahre eine interessante empirische Studie vor, in der er das Kulturverständnis von Menschen aus unterschiedlichen Generationen untersuchte (Göschel 1991). Er kam zu dem Ergebnis, dass sich im Zehnjahresabstand das Verständnis von Kultur deutlich ändert. Daraus ergibt sich zwangsläufig ein sich entsprechend veränderndes Verständnis von Kulturpolitik und ihren Aufgaben. So identifiziert Albrecht Göschel in der Zeit nach dem Zweiten Weltkrieg eine konservative Kulturpolitik (,Kulturpflege“) des Bewahrens, was für das Theater eine textnahe Umsetzung der klassischen Literatur bedeutet. In den 1960er Jahren entwickelt sich ein emanzipatorischer Ansatz der Aufklärung und der Soziokultur, bei dem die demokratische Forderung einer „Kultur für alle“ eine zentrale Rolle spielte. Es folgte eine Generation, die wesentlich durch sogenannte Humandienstleister (Psychologen, Pädagogen etc.) geprägt ist und die ihren individuellen Lebensstil in den Vordergrund stellte.

Auch wenn diese These einer Generationenabfolge im Zehnjahresabstand zu grob erscheint, so weist sie doch darauf hin, dass mit der gesellschaftlichen Veränderung auch eine deutliche Veränderung gesellschaftlicher Diskurse und damit auch eine Veränderung der Verständnisweisen von Kultur und Kulturpolitik einhergeht. Dies lässt sich zum einen daran erkennen, dass Leitformeln und Slogans, mit denen man die eigene Arbeit legitimieren und begründen will, ebenfalls einem Wandel unterliegen (Fuchs 2011b). So wird in den 1960er und 1970er Jahren das Konzept einer konservativen und bloß konservierenden „Kulturpflege" obsolet und von dem Leitbegriff der Emanzipation verdrängt. Spätere Generationen können wiederum mit dem Emanzipationsbegriff wenig anfangen. 
Seither ist die Frage nach einem allgemein akzeptierten Leitbegriff einer der Streitpunkte in kulturpolitischen Debatten. Ich komme bei der Thematisierung der Begründungsmöglichkeiten von Kulturpolitik darauf zurück.

Man kann geradezu eine „Theorie“ solcher Leitformen entwickeln, in der man diesen Wandlungsprozess von Begründungsmustern untersucht (Fuchs 2011b). Dabei wird man feststellen, dass Leitformeln auf gesellschaftliche Entwicklungstendenzen und vor allen Dingen auf Problemlagen reagieren. Wenn heute eine wichtige Leitformel „Teilhabe“ ist, so hat das mit dem Problem zu tun, dass wichtige Bevölkerungsgruppen zunehmend von ökonomischer, sozialer, politischer und kultureller Teilhabe ausgeschlossen werden (Maedler 2008). Wenn heute Begriffe wie Vielfalt und Diversität bei der Begründung und Legitimation künstlerischer Aktivitäten häufig zu finden sind, so hat dies offensichtlich damit zu tun, dass unsere Gesellschaft sich schwer tut damit, die wachsende ethnische Vielfalt in unserer Gesellschaft zu akzeptieren.

Solche Leitformeln haben also die Funktion der Begründung, sie reflektieren allerdings auch gesellschaftliche Verhältnisse und Problemlagen und sie suggerieren, dass die jeweiligen kulturellen Angebote in der Lage sind, etwas zur Behebung der identifizierten Problemlagen zu leisten. Es steckt also eine Zuschreibung von Funktionen und Wirkungen der jeweiligen künstlerischen Aktivität hinter der Verwendung entsprechender Leitformeln und Slogans. Beispiele für diese Behauptung sind am Anfang dieses Textes gegeben worden.

Man kann dabei unterschiedliche Formen der Begründung der Notwendigkeit von Kunst und speziell von Theater unterscheiden, wobei in der Praxis meist mehrere dieser Begründungsmöglichkeiten genutzt werden. Insbesondere kann man die folgenden Formen der Begründung identifizieren:

- eine anthropologische Begründung: Theater gehört zum Menschsein dazu

- eine pädagogische Begründung: Theater bildet zur Humanität

- eine gesellschaftspolitische Begründung: durch Theater entsteht Integration und Zusammenhalt

- eine kunsttheoretische Begründung: Theater ist eine autonome Kunstform, dies oft in Verbindung mit

- einer rechtlichen Begründung: es gibt die Kunstfreiheitsgarantie, die durch entsprechende Urteile des Bundesverfassungsgerichtes auch eine Aufforderung zur Förderung der Kunst durch den Staat beinhaltet; zudem kann man sich hier auf das Menschenrecht auf kulturelle Teilhabe beziehen 
- eine ökonomische Begründung: eine solche Argumentation (Stichwort: Umwegrentabilität und Arbeitsmarkt Kultur, heute: Förderung der Kreativität) ist umstritten, wird aber gerade auf kommunaler Ebene immer noch gerne genutzt

- eine stadtpolitische Begründung: Steigerung der Attraktivität der Stadt für Tourist*innen und Gewerbeansiedlungen durch ein attraktives Theater.

Es liegt auf der Hand, dass jede dieser Begründungen zu einer bestimmten Verständnisweise von Kultur- und Theaterpolitik gehört. So kann man eine ganze Reihe solcher Verständnisweisen unterscheiden, nämlich unter Kultur- und Theaterpolitik unter anderem zu verstehen als: Stadtentwicklungs-, Lebensstil-, Wirtschafts-, Gesellschafts-, Bildungs-, Kunst-, Macht-, Mentalitätspolitik oder Politik der Teilhabe und Vielfalt, der Distinktion oder der Anerkennung.

Die individuelle Auswahl aus diesen Verständnisweisen hängt sowohl von persönlichen Überzeugungen, aber auch davon ab, welche gesellschaftlichen Diskurse gerade relevant sind. Denn die verwendeten Leitformeln und Slogans, die mit solchen Verständnisweisen verbunden sind, haben nicht nur die Aufgabe, eigene Überzeugungen über die Rolle von Theater für den Menschen und die Gesellschaft auszudrücken: Sie zielen auch auf die Akzeptanz durch andere und damit auf die Bereitschaft, eine Förderung nicht zu versagen.

Das Problem, das sich hierbei ergibt, liegt in der Ausdifferenzierung der Gesellschaft in unterschiedliche Milieus und Lebensstilgruppen. Spätestens seit den Studien von Pierre Bourdieu (1987) kennt man den engen Zusammenhang von Milieu und ästhetischer Präferenz. Eine wichtige Erkenntnis besteht darin, dass die Künste zwar durchaus ein Mittel der Integration sind, dies allerdings nur im Rahmen der eigene Lebensstilgruppe. Im Hinblick auf andere Lebensstilgruppen sind sie Medien der Distinktion. Daher ist es schwer, wenn nicht gar unmöglich, quer durch unterschiedliche Lebensstilgruppen für die gesamte Gesellschaft nicht nur Begründungsmuster, sondern auch entsprechende Angebote zu finden, die auf eine generelle Akzeptanz stoßen können. Dies ist in der kulturpolitischen Debatte natürlich nicht neu. So hatte schon der Soziologe Gerhard Schulze (1992) in seinem damaligen Bestseller zur „Erlebnisgesellschaft“ nicht nur unterschiedliche gesellschaftliche Szenen unterschieden (Hochkulturszene, neue Kulturszene, Kulturladenszene, Kneipenszene), er hat auch unterschiedliche kulturpolitische Leitmotive, also Ziele der Kulturpolitik, identifiziert, die eine große Ähnlichkeit mit den oben vorgestellten Forschungsergebnissen von Albrecht Göschel haben, nämlich das Hochkulturmotiv (von 1945 bis in die 1960er Jahre hinein), das Demokratisierungsmotiv (so etwa bei Hilmar Hoffmann und Hermann Glaser), das soziokulturelle Motiv (mit dem Ziel der Verbesserung 
der Kommunikation) und nicht zuletzt das Ökonomiemotiv. Nicht zu vergessen ist das meist nicht angesprochene Motiv der Selbsterhaltung der Einrichtung. Denn ein Problem in der Kultur- und Bildungspolitik besteht darin, dass zwar oft genug damit argumentiert wird, welche wichtige Rolle die jeweiligen Angebote für andere Menschen oder die Gesellschaft als Ganzes spielen, nicht explizit erwähnt wird allerdings, dass es dabei auch um die Erhaltung der eigenen Arbeitsplätze geht. Das ist zwar legitim und notwendig, da man Institutionen und Strukturen braucht, damit Angebote realisiert werden können. Doch schmälert dieses Eigeninteresse einen gelegentlich zu findenden altruistischen Duktus in Positionspapieren und Forderungskatalogen (siehe hierzu das immer noch relevante Kapitel „Paradoxien der Kulturpolitik“ in Schulze 1992, S. 495 ff.).

Neben der Unterschiedlichkeit der verschiedenen Funktionszuschreibungen, die in den Slogans und Begründungsmustern in der Theater- und Kulturpolitik zugrunde liegen, dürfte ein Hauptproblem darin bestehen, dass man es mit nicht aufzuhebenden Widersprüchen in diesem Feld zu tun hat. Bereits Gerhard Schulze sprach - wie erwähnt - von „Paradoxien der Kulturpolitik“. So gibt es wie angedeutet - nicht bloß unterschiedliche Verständnisweisen von Kulturpolitik je nach politischer Ebene, es gibt auch deutliche Unterschiede im Hinblick auf die anzustrebenden Ziele: Kunstfreiheit und/oder Wirtschaftlichkeit, Qualität und Quantität, die Kritik neoliberaler und antidemokratischer Tendenzen in der Gesellschaft bei gleichzeitiger Beibehaltung autoritärer Binnenstrukturen, den Anspruch Kultur für alle zu realisieren in einer Gesellschaft, die sich gerade durch ästhetische Ausdrucksformen wesentlich ausdifferenziert, die Unterschiede zwischen kulturellen Infrastrukturen in der Stadt und auf dem Land, die Herausforderung gesellschaftlicher Entwicklungen wie Digitalisierung und Globalisierung.

In diesem vorliegenden Beitrag sollen und können keine Vorschläge für die Lösung dieser Widersprüche angeboten werden. Es scheint mir allerdings eine zentrale Aufgabe des Theaters darin zu bestehen, diese Widersprüche in der Gesellschaft zu reflektieren, aber auch in einem zu intensivierenden Selbstreflexionsprozess zur Kenntnis zu nehmen, ob und wie man diese Widersprüche in der eigenen Arbeit auch immer wieder reproduziert.

\section{Literatur}

Arbeitsgruppe ,Zukunft von Theaters und Oper in Deutschland. 2004. Zwischenbericht. In Jahrbuch für Kulturpolitik 2004: Thema: Theaterdebatte, Hrsg. Institut für Kulturpolitik der Kulturpolitischen Gesellschaft, 343 - 352. Essen: Klartext. 
Behrens, F., R. Heinze, J. Hilbert, S. Stöbe, und E. Walsken, Hrsg. 1995. Den Staat neu denken. Berlin: Sigma.

Berg, S. 2017. Über das Theater und seinen Wunsch, politisch zu sein. Vortrag bei einer Tagung der Intendantengruppe des Deutschen Bühnenvereins am 9.12. 2017 in Hofgeismar. Nachtkritik. https://nachtkritik.de/index.php?option=com_content\&view=article\& id=14789: vortrag-sibylle-berg\&catid=101\&ltemid=84. Zugegriffen: 15. Juni 2020.

Bourdieu, Pierre. 1987. Die feinen Unterschiede. Frankfurt a. M.: Suhrkamp.

Brodocz, A., und G. Schaal, Hrsg. 2017. Politische Theorien der Gegenwart I-III. Stuttgart: UTB.

Deutsche UNESCO-Kommission, Hrsg. 2007. Kulturelle Vielfalt - Unser gemeinsamer Reichtum. Bonn: DUK.

Deutscher Bühnenverein, Hrsg. 2003. Muss Theater sein? Köln: DBV.

Deutscher Bundestag, Hrsg. 2008. Kultur in Deutschland. Regensburg: conbrio.

Fischer-Lichte, Erika. 1993. Kurze Geschichte des deutschen Theaters. Tübingen: Francke.

Fuchs, Max. 1998. Kulturpolitik als gesellschaftliche Aufgabe. Wiesbaden: Westdeutscher Verlag.

Fuchs, Max. 2007. Kulturpolitik. Wiesbaden: VS.

Fuchs, Max. 2011a. Leitformeln und Slogans in der Kulturpolitik. Wiesbaden: VS.

Fuchs, Max. 2011b. Kunst als kulturelle Praxis. München: Kopaed.

Glaser, Hermann, und K. H. Stahl. 1974/1983. Bürgerrecht Kultur. 2. Aufl. Frankfurt am Main u. a.: Ullstein.

Göschel, Albrecht. 1991. Die Ungleichzeitigkeit in der Kultur. Stuttgart: Kohlhammer.

Hoffmann, Hilmar. 1979. Kultur für alle. Frankfurt a. M.: S. Fischer.

Institut für Kulturpolitik der Kulturpolitischen Gesellschaft, Hrsg. 2004. Jahrbuch für Kulturpolitik 2004 - Thema: Theaterdebatte. Essen: Klartext.

Jann, Werner. 1998. Neues Steuerungsmodell. In Handbuch zur Verwaltungsreform, Hrsg. B. Blanke, 70-79. Opladen: Leske und Budrich.

Klein, Armin. 2005. Kulturpolitik. Eine Einführung. Wiesbaden: Springer VS.

Kommunale Gemeinschaftsstelle für Verwaltungsvereinfachung. 1989. Führung und Steuerung des Theaters. Köln: KGSt.

Maedler, Jens, Hrsg. 2008. TeileHabeNichtse. München: Kopaed.

Nipperdey, Thomas. 1998. Deutsche Geschichte 1866 - 1918, Band 2: Machtstaat vor Demokratie. München: Beck.

Rau, Johannes. 2004 „Bündnis für Theater“. In Jahrbuch für Kulturpolitik 2004. Thema: Theaterdebatte, Hrsg. Institut für Kulturpolitik, 37-43. Essen: Verlag Klartext.

Scheytt, Oliver. 2008. Kulturstaat Deutschland. Bielefeld: transcript.

Scheytt, O., S. Raskop und G. Willems, Hrsg. 2016. Die Kulturimmobilie. Bielefeld: transcript.

Schulze, Gerhard. 1992. Die Erlebnisgesellschaft. Frankfurt a. M.: Campus.

Schwencke, Olaf, Hrsg. 2006. Das Europa der Kulturen. Essen: Klartext.

Wagner, Bernd. 2009. Fürstenhof und Bürgergesellschaft. Essen: Klartext. 
Open Access Dieses Kapitel wird unter der Creative Commons Namensnennung 4.0 International Lizenz (http://creativecommons.org/licenses/by/4.0/deed.de) veröffentlicht, welche die Nutzung, Vervielfältigung, Bearbeitung, Verbreitung und Wiedergabe in jeglichem Medium und Format erlaubt, sofern Sie den/die ursprünglichen Autor(en) und die Quelle ordnungsgemäß nennen, einen Link zur Creative Commons Lizenz beifügen und angeben, ob Änderungen vorgenommen wurden.

Die in diesem Kapitel enthaltenen Bilder und sonstiges Drittmaterial unterliegen ebenfalls der genannten Creative Commons Lizenz, sofern sich aus der Abbildungslegende nichts anderes ergibt. Sofern das betreffende Material nicht unter der genannten Creative Commons Lizenz steht und die betreffende Handlung nicht nach gesetzlichen Vorschriften erlaubt ist, ist für die oben aufgeführten Weiterverwendungen des Materials die Einwilligung des jeweiligen Rechteinhabers einzuholen.

(c) (1) 